\title{
A Mouse Mammary Gland Involution mRNA Signature Identifies Biological Pathways Potentially Associated with Breast Cancer Metastasis
}

\author{
Torsten Stein • Nathan Salomonis • \\ Dimitry S. A. Nuyten - Marc J. van de Vijver • \\ Barry A. Gusterson
}

Published online: 28 May 2009

(C) Springer Science + Business Media, LLC 2009

\section{Erratum to: J Mammary Gland Biol Neoplasia DOI 10.1007/s10911-009-9120-1}

The original version of this article unfortunately contained a mistake. The presentation of Tables 1 and 2 were incorrect. The corrected tables are given below.

The Publishers apologise for the errors that exist in the tables in this article. The errors were introduced by the publisher during the publication process at a stage that was too late to be corrected.

The online version of the original article can be found at http://dx.doi. org/10.1007/s10911-009-9120-1.

\footnotetext{
T. Stein · B. A. Gusterson

Division of Cancer Sciences and Molecular Pathology,

Section of Gene Regulation and Mechanisms of Disease,

Western Infirmary, University of Glasgow,

Glasgow G11 6NT, UK

\section{N. Salomonis}

Gladstone Institute of Cardiovascular Disease,

1650 Owens Street,

San Francisco, CA 94158, USA

\section{N. Salomonis}

Pharmaceutical Sciences and Pharmacogenomics Graduate

Program, University of California,

513 Parnassus Avenue,

San Francisco, CA 94143, USA
}

\section{S. A. Nuyten}

Department of Radiation Oncology,

The Netherlands Cancer Institute,

Plesmanlaan 121,

Amsterdam 1066 CX, The Netherlands

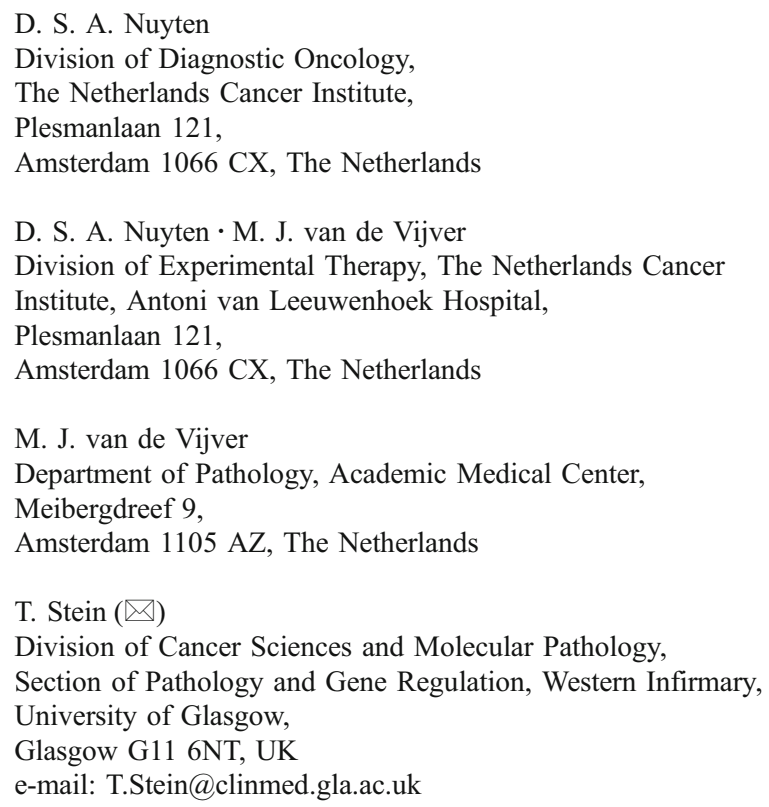


Table 1 Cox regression tables for involution signatures (Survival).

\begin{tabular}{|c|c|c|c|c|}
\hline \multirow[t]{2}{*}{ Variable } & \multirow[t]{2}{*}{ Significance } & \multirow[t]{2}{*}{ Hazard Ratio for Death } & \multicolumn{2}{|c|}{$95.0 \%$ CI for $\mathrm{HR}$} \\
\hline & & & Lower & Upper \\
\hline \multicolumn{5}{|c|}{ A: Clinical risk factors and Inv3 signature: survival (Cox regression) } \\
\hline Diameter T2 $(>2 \mathrm{~cm})$ vs T1 $(\leq 2 \mathrm{~cm})$ & 0.07 & 1.48 & 0.98 & 2.26 \\
\hline Lymph node positive vs negative & 0.34 & 1.35 & 0.73 & 2.51 \\
\hline Age above 40 versus 40 and below & 0.14 & 0.71 & 0.45 & 1.11 \\
\hline ER positive vs negative & 0.15 & 0.71 & 0.45 & 1.13 \\
\hline Grade 3 vs 2 vs 1 & 0.01 & 1.61 & 1.15 & 2.24 \\
\hline Chemotherapy, yes vs no & 0.48 & 0.80 & 0.43 & 1.48 \\
\hline Hormonal therapy, yes vs no & 0.43 & 0.74 & 0.35 & 1.56 \\
\hline Inv3 Signature, poor vs good & 0.003 & 2.36 & 1.33 & 4.17 \\
\hline \multicolumn{5}{|c|}{ B: Clinical risk factors and C5 signature: survival (Cox regression) } \\
\hline Diameter T2 $(>2 \mathrm{~cm})$ vs T1 $(\leq 2 \mathrm{~cm})$ & 0.07 & 1.48 & 0.98 & 2.26 \\
\hline Lymph node positive vs negative & 0.19 & 1.52 & 0.81 & 2.85 \\
\hline Age above 40 versus 40 and below & 0.08 & 0.67 & 0.43 & 1.05 \\
\hline ER positive vs negative & 0.38 & 0.82 & 0.51 & 1.29 \\
\hline Grade 3 vs 2 vs 1 & 0.01 & 1.59 & 1.14 & 2.22 \\
\hline Chemotherapy, yes vs no & 0.25 & 0.69 & 0.37 & 1.30 \\
\hline Hormonal therapy, yes vs no & 0.44 & 0.75 & 0.35 & 1.57 \\
\hline C5 Signature, poor vs good & $<10^{-3}$ & 2.94 & 1.66 & 5.20 \\
\hline \multicolumn{5}{|c|}{ C: Clinical risk factors and Inv3/C5 signature: survival (Cox regression) } \\
\hline Diameter T2 $(>2 \mathrm{~cm})$ vs T1 $(\leq 2 \mathrm{~cm})$ & 0.12 & 1.39 & 0.91 & 2.12 \\
\hline Lymph node positive vs negative & 0.22 & 1.49 & 0.79 & 2.84 \\
\hline Age above 40 versus 40 and below & 0.07 & 0.66 & 0.42 & 1.04 \\
\hline ER positive vs negative & 0.19 & 0.74 & 0.47 & 1.16 \\
\hline Grade 3 vs 2 vs 1 & 0.001 & 1.72 & 1.24 & 2.37 \\
\hline Chemotherapy, yes vs no & 0.39 & 0.76 & 0.40 & 1.43 \\
\hline Hormonal therapy, yes vs no & 0.43 & 0.74 & 0.35 & 1.56 \\
\hline Inv3/C5 Signature, poor vs good & $<10^{-3}$ & 3.13 & 1.69 & 5.80 \\
\hline
\end{tabular}


Table 2 Cox regression tables for involution signatures (Metastasis).

\begin{tabular}{|c|c|c|c|c|}
\hline \multirow[t]{2}{*}{ Variable } & \multirow[t]{2}{*}{ Significance } & Hazard Ratio for Metastasis & \multicolumn{2}{|c|}{$95.0 \%$ CI for HR } \\
\hline & & & Lower & Upper \\
\hline \multicolumn{5}{|c|}{ A: Clinical risk factors and Inv3 signature: metastasis (Cox regression) } \\
\hline Diameter T2 $(>2 \mathrm{~cm})$ vs T1 $(\leq 2 \mathrm{~cm})$ & 0.02 & 1.62 & 1.10 & 2.39 \\
\hline Lymph node positive vs negative & 0.17 & 1.49 & 0.84 & 2.62 \\
\hline Age above 40 versus 40 and below & 0.06 & 0.67 & 0.44 & 1.02 \\
\hline ER positive vs negative & 0.53 & 1.16 & 0.73 & 1.84 \\
\hline Grade 3 vs 2 vs 1 & 0.04 & 1.36 & 1.02 & 1.81 \\
\hline Chemotherapy, yes vs no & 0.22 & 0.70 & 0.40 & 1.23 \\
\hline Hormonal therapy, yes vs no & 0.19 & 0.64 & 0.33 & 1.25 \\
\hline Inv3 Signature, poor vs good & $<10^{-3}$ & 2.77 & 1.66 & 4.64 \\
\hline \multicolumn{5}{|c|}{ B: Clinical risk factors and C5 signature: metastasis (Cox regression) } \\
\hline Diameter T2 $(>2 \mathrm{~cm})$ vs T1 $(\leq 2 \mathrm{~cm})$ & 0.01 & 1.63 & 1.11 & 2.41 \\
\hline Lymph node positive vs negative & 0.06 & 1.74 & 0.98 & 3.09 \\
\hline Age above 40 versus 40 and below & 0.02 & 0.62 & 0.41 & 0.94 \\
\hline ER positive vs negative & 0.29 & 1.29 & 0.81 & 2.05 \\
\hline Grade 3 vs 2 vs 1 & 0.03 & 1.39 & 1.04 & 1.85 \\
\hline Chemotherapy, yes vs no & 0.08 & 0.60 & 0.34 & 1.07 \\
\hline Hormonal therapy, yes vs no & 0.19 & 0.64 & 0.33 & 1.26 \\
\hline C5 Signature, poor vs good & $<10^{-4}$ & 2.80 & 1.71 & 4.58 \\
\hline \multicolumn{5}{|c|}{ C: Clinical risk factors and Inv3/C5 signature: metastasis (Cox regression) } \\
\hline Diameter T2 $(>2 \mathrm{~cm})$ vs T1 $(\leq 2 \mathrm{~cm})$ & 0.03 & 1.54 & 1.04 & 2.27 \\
\hline Lymph node positive vs negative & 0.08 & 1.67 & 0.93 & 2.98 \\
\hline Age above 40 versus 40 and below & 0.03 & 0.63 & 0.42 & 0.95 \\
\hline ER positive vs negative & 0.57 & 1.14 & 0.72 & 1.80 \\
\hline Grade 3 vs 2 vs 1 & 0.004 & 1.51 & 1.14 & 2.00 \\
\hline Chemotherapy, yes vs no & 0.19 & 0.68 & 0.38 & 1.20 \\
\hline Hormonal therapy, yes vs no & 0.22 & 0.66 & 0.33 & 1.29 \\
\hline Inv3/C5 Signature, poor vs good & $<10^{-3}$ & 2.48 & 1.50 & 4.11 \\
\hline
\end{tabular}

